\title{
Retinal expression, regulation, and functional bioactivity of prostacyclin-stimulating factor
}

\author{
Yasuaki Hata, ${ }^{1}$ Allen Clermont,${ }^{1,2}$ Teruaki Yamauchi, ${ }^{1}$ Eric A. Pierce, ${ }^{3}$ Izumi Suzuma, ${ }^{1}$ \\ Hiroyuki Kagokawa, ${ }^{1}$ Hiroshi Yoshikawa, ${ }^{4}$ Gregory S. Robinson, ${ }^{6}$ Tatsuro Ishibashi, ${ }^{4}$ \\ Toshihiko Hashimoto, ${ }^{5}$ Fumio Umeda, ${ }^{5}$ Sven E. Bursell, ${ }^{1,2}$ and Lloyd Paul Aiello ${ }^{1,2,7}$
}

\author{
${ }^{1}$ Research Division, and \\ ${ }^{2}$ Beetham Eye Institute, Joslin Diabetes Center, Boston, Massachusetts, USA \\ ${ }^{3}$ F.M. Kirby Center for Molecular Ophthalmology, Scheie Eye Institute, University of Pennsylvania, Philadelphia, \\ Pennsylvania, USA \\ ${ }^{4}$ Department of Ophthalmology, and \\ ${ }^{5}$ Department of Internal Medicine, Kyushu University, Faculty of Medicine, Fukuoka, Japan \\ ${ }^{6}$ Hybridon Inc., Cambridge, Massachusetts, USA \\ ${ }^{7}$ Department of Ophthalmology, Harvard Medical School, Boston, Massachusetts, USA
}

Address correspondence to: Lloyd Paul Aiello, Joslin Diabetes Center, One Joslin Place, Boston, Massachusetts 02215, USA. Phone: (617) 732-2427; Fax: (617) 735-1960; E-mail: LPAiello@Joslin.Harvard.Edu.

Received for publication September 2, 1999, and accepted in revised form July 14, 2000.

Prostacyclin-stimulating factor (PSF) acts on vascular endothelial cells to stimulate the synthesis of the
vasodilatory molecule prostacyclin (PGI2). We have examined the expression, regulation, and hemody-
namic bioactivity of PSF both in whole retina and in cultured cells derived from this tissue. PSF was
expressed in all retinal cell types examined in vitro, but immunohistochemical analysis revealed PSF
mainly associated with retinal vessels. PSF expression was constitutive in retinal pericytes (RPCs) but
could be modulated in bovine retinal capillary endothelial cells (RECs) by cell confluency, hypoxia, serum
starvation, high glucose concentrations, or inversely by soluble factors present in early vs. late retinopa-
thy, such as TGF- $\beta$, VEGF, or bFGF. In addition, RPC-conditioned media dramatically increased REC
PGI 2 production, a response inhibited by blocking PSF with a specific antisense oligodeoxynucleotide
(ODN). In vivo, PGI2 increased retinal blood flow (RBF) in control and diabetic animals. Furthermore,
the early drop in RBF during the initial weeks after inducing diabetes in rats, as well as the later increase
in RBF, both correlated with levels of retinal PSF. RBF also responded to treatment with RPC-conditioned
media, and this effect could be partially blocked using the antisense PSF ODN. We conclude that PSF
expressed by ocular cells can induce PGI2, retinal vascular dilation, and increased retinal blood flow, and
that alterations in retinal PSF expression may explain the biphasic changes in RBF observed in diabetes. that alterations in retinal PSF expression may explain the biphasic changes in RBF observed in diabetes.
J. Clin. Invest. 106:541-550 (2000).

\section{Introduction}

Diabetes is associated with an early reduction in retinal blood flow (RBF) followed by gradual increase in RBF as diabetic retinopathy progresses (1-3). The changes in $\mathrm{RBF}$ may contribute to the progression of retinal pathology that ultimately leads to severe, irreversible vision loss. However, the mechanisms underlying this biphasic change in RBF are not well understood.

Prostacyclin-stimulating factor (PSF), a recently identified molecule with $\mathrm{NH}_{2}$-terminal amino acid sequence homology with IGFBP-4 (4), was originally purified and cloned from human diploid fibroblast cells (4). PSF is a 31-kDa acid-labile, anionic, and heparin-binding molecule. The single-copy gene in humans is comprised of five exons and four introns (5). There are seven Sp1 transcription-factor binding sites within the initial $300 \mathrm{bp}$ of the upstream promoter region.

Numerous tissues, including rat kidney, lung, aorta, brain, liver, skeletal muscle, smooth muscle, fat, and human coronary artery, aorta, vaso vasorum, and fibroblasts express PSF RNA and protein (4-11). In streptozotocin-induced diabetic rats, kidney PSF mRNA expression is decreased and less PSF immunohistochemical staining is found in the renal arteries (6, 8). Similar reductions occur in the diabetic and atherosclerotic human coronary artery (7). However, no changes in PSF RNA expression or immunohistochemical staining have been observed in the lungs of diabetic animals, and PSF mRNA expression is increased in colon cancer and adenocarcinoma cell lines (12). The biological significance of these changes is unknown.

The principle known action of PSF is to stimulate prostacyclin (PGI2) production by endothelial cells. PSF concentrations as low as $10 \mathrm{ng} / \mathrm{mL}$ stimulate PGI2 in bovine aortic endothelial cells within $30 \mathrm{~min}-$ utes, with an eightfold increase observed at $25 \mathrm{ng} / \mathrm{mL}$ after 1 hour (4). Although numerous substances including arachidonic acid, phorbol ester (13), PDGF (14), Il-1 (15), TNF (16), EGF (17), and TGF- $\beta$ (18) stimulate PGI2, stimulation of PGI2 production by PSF is thought to be mediated primarily by the arachidonic acid cascade (4). 
Prostacyclin is a vasoactive prostaglandin produced by vascular cells that induce vasodilation in vivo at physiologic concentrations $(19,20)$. PGI2 is also reported to increase vascular permeability $(21,22)$, promote pericyte relaxation (20), and mediate nitric oxideinduced (NO-induced) ocular vasorelaxation (19). Elevated glucose concentrations stimulate PGI2 secretion in human retinal pericytes (RPCs) (23), and acutely elevated blood glucose increases retinal blood flow (1). Thus, changes in PSF activity or expression induced by diabetes may alter retinal hemodynamics. Little is known, however, regarding the expression, regulation, or activity of PSF in the retina.

In this study we evaluated retinal expression of PSF in vitro and in vivo and have explored its effects on retinal hemodynamics under normal and diabetic conditions. We observe that PSF is associated with the retinal vasculature in vivo and is expressed by numerous ocular cell types in vitro. PSF increases PGI2 production in retinal endothelial cells, resulting in vasodilation and increased RBF in vivo. Retinal PSF expression is reduced during early diabetes and increased in more advanced diabetes. Furthermore, PSF is tightly regulated by various factors associated with diabetic retinopathy, with decreased expression induced by factors predominating in early retinopathy and increased expression associated with factors involved in advancing retinopathy. These results imply that PSF may serve an important role in the diabetic retina and suggest a mechanism to account for the biphasic RBF abnormalities classically associated with progressing diabetic retinopathy.

\section{Methods}

Cell culture. Primary cultures of bovine retinal capillary endothelial cells (RECs), RPCs, retinal pigment epithelial cells (RPEs), aortic endothelial cells (AECs), and smooth muscle cells (SMCs) were isolated from fresh slaughterhouse tissues as described previously (24). RECs were cultured in endothelial basal medium (CLONETICS Corp., San Diego, California, USA) with 10\% plasma-derived horse serum (Sigma Chemical Co., St. Louis, Missouri, USA), heparin $(50 \mathrm{mg} / \mathrm{L})$, and $50 \mu \mathrm{g} / \mathrm{mL}$ of endothelial cell-growth factor (Roche, Indianapolis, Indiana, USA). RPCs were cultured in DMEM with 20\% FBS (HyClone Laboratories, Logan, Utah, USA). RPEs, AECs, and SMCs were cultured in DMEM with $10 \%$ calf serum (Life Technologies Inc., Gaithersburg, Maryland, USA). All cells were cultured at $37^{\circ} \mathrm{C}$ in $5 \% \mathrm{CO}_{2}, 95 \%$ air, and media were changed every $2-3$ days.

Immunohistochemistry. Rat eyes were enucleated and immediately fixed in 4\% paraformaldehyde in $0.1 \mathrm{M}$ phosphate buffer ( $\mathrm{pH} 7.4$ ) for 2 hours. Eyes were gradually dehydrated in ethanol, embedded in paraffin, and $3-\mu \mathrm{m}$-thick sections were deparaffinized and incubated for 1 hour in 0.01 M PBS containing 1\% dry milk. After incubation overnight with anti-PSF polyclonal $\mathrm{Ab}$ (7) at $4{ }^{\circ} \mathrm{C}$, sections were washed for 15 minutes with PBS. Immunoperoxidase detection was performed using avidin-biotin-complex method with aminoethylcarbizole as the red chromogen. Polyclonal anti-von Willebrand factor (Sigma Chemical Co.) was used to identify retinal vessels. Negative control substituted rabbit nonimmune IgG for the primary $\mathrm{Ab}$.

Northern blot analysis. Total RNA samples were isolated from cells using acid guanidinium thiocyanate-phenol-chloroform-extraction method (25) and subjected to Northern blot analysis as described previously. Radioactive PSF and TGF- $\beta$ cDNA probes $(9,26)$ were generated using Amersham Multiprime labeling kits and ${ }^{32} \mathrm{P}-\mathrm{dCTP}$ (NEN Life Science Products, Boston, Massachusetts, USA). Quantitation of Northern blots was performed using a computing PhosphorImager with ImageQuant software analysis (Molecular Dynamics, Sunnyvale, California, USA). Lane-loading differences were normalized by rehybridization with radiolabeled 36B4 cDNA probe (27).

Transfection and preparation of pericyte-conditioned media. A phosphorothioate-modified antisense oligodeoxynucleotide (5'-AGG AGC AGG GCG CGC AGC GA-3') directed against PSF base pairs 35-54 from transcription start site, its sense control (5'-TC GCT GCG CGC CCT GCT CCT- $3^{\prime}$ ), and a randomized, equal-length oligodeoxynucleotide were commercially synthesized (Oligos, Etc., Wilsonville, Oregon, USA). The day before transfection, pericytes were seeded in six multiwell plates in normal growth medium. The day of transfection, subconfluent pericytes were washed with fresh DMEM and transfected with $0.5 \mu \mathrm{M}$ oligodeoxynucleotide using Lipofectamine reagent (Life Technologies Inc.) as instructed by the manufacturer. After 6 hours, medium was changed to fresh DMEM without serum. Twenty-four hours after initial transfection, supernatants were collected and subjected to Western blot analysis or intravitreal injection. For intravitreal injection or use as conditioned media, samples were concentrated 25 -fold, using a centrifugal filter device (Millipore, Bedford, Massachusetts, USA) with a 10,000-MW cutoff.

Western blot analysis. Equal volumes of conditioned media were subjected to $15 \%$ SDS-PAGE, and proteins were transferred to nitrocellulose filters (BioRad, Hercules, California, USA). The blots were incubated with primary Ab's followed by horseradish peroxidase-conjugated secondary Ab (Amersham Corp). Visualization was performed using Amersham enhanced chemiluminescence (ECL) detection system per the manufacturer's instructions.

Measurement of PGI2 synthesis. Concentration of the stable metabolite of prostacyclin, 6-keto-prostaglandin $\mathrm{F} 1_{\alpha}$ (6-keto-PGF1 $\alpha$ ), in the conditioned media was measured by radioimmunoassay (Amersham Corp.) per the manufacturer's instructions.

Intravitreal injection of PGI2. PGI2 (Sigma Chemical Co.) was dissolved in $100 \%$ ethanol and then diluted in saline containing 2.5\% Emulphor, resulting in $10 \mu \mathrm{M}$ PGI2 stock ( $0.3 \%$ ethanol). PGI2 stock $(10 \mu \mathrm{L})$ was injected intravitreally ( $1 \mu \mathrm{M}$ final concentration) and retinal hemodynamic parameters were measured. 
a

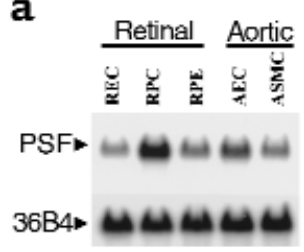

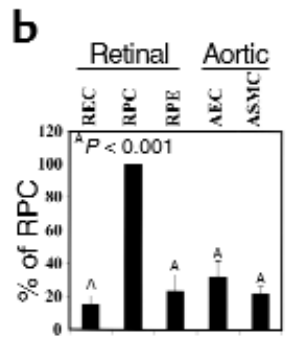

Figure 1

Retinal cells express PSF mRNA in vitro. Confluent cultures of bovine microvascular RECs, RPCs, RPEs, AECs, and ASMCs were evaluated for PSF mRNA expression by Northern blot analysis using a randomprimed PSF cDNA probe (a). Northern blots were stripped and reprobed with control 36B4 cDNA as a lane-loading control. Quantitation of multiple experiments after normalization to the 36B4 control signal is also presented (b). ${ }^{A} P<0.001$.

Oxygen-induced retinopathy in the neonatal mouse. Oxygeninduced retinopathy was elicited in neonatal mice by standard techniques $(28,29)$. Retinal neovascularization similar to proliferative diabetic retinopathy developed in $100 \%$ of the animals by postnatal day 17 (P17).

Retinal bemodynamic studies. The video fluorescein angiography (VFA) system used for these studies has been described previously (30-35). Retinal segmental mean circulation time (MCT) and RBF for each rat was derived as described previously (30-38).

Animals. Male Sprague-Dawley rats (Taconic Farms, Germantown, New York, USA) weighing approximately $250 \mathrm{~g}$ were cared for in accordance with the Association for Research in Vision and Ophthalmology Statement for the Use of Animals in Ophthalmic and Vision Research. Diabetes was induced by intraperitoneal injection of streptozotocin (STZ; Sigma Chemical Co.) at 65 $\mathrm{mg} / \mathrm{kg}$ body weight in $10 \mathrm{mM}$ citrate buffer, $\mathrm{pH} 4.5$, after a 12-hour fast. Diabetes was confirmed with blood glucose measurements 24 hours after STZ injection. The rats were housed under standard conditions with one rat per cage with free access to water and food. Blood glucose levels and body weights were monitored every other day. Blood glucose levels did not exceed $400 \mathrm{mg} / \mathrm{dL}$ in the diabetic animals, and all diabetic rats gained weight.

Immediately before VFA measurements, each rat was anesthetized with $0.1 \mathrm{mg} / \mathrm{kg}$ sodium amobarbital (Lilly Research Laboratories, Indianapolis, Indiana, USA) injected intraperitoneally, and the pupil of the left eye was dilated using 1\% tropicamide (Mydriacyl; Alcon, Fort Worth, Texas, USA). Base-line angiograms $(5-\mu \mathrm{L}$ bolus of fluorescein dye) were recorded from each rat. Rats then underwent an intravitreal injection and repeat angiograms, as indicated. Intravitreal injections were performed as described previously (39).

Statistical analysis. All experiments were repeated at least three times with similar findings, and results are expressed as mean plus or minus SD unless otherwise noted. The unpaired $t$ test was used for single comparisons of groups with equal variance and normal distribution. The Mann-Whitney test was used if the groups were of unequal variance or non-normal distribution. ANOVA with Tukey's test was used for multiple comparisons of data with equal variance and normal distribution, whereas ANOVA with Dunnett's method of multiple-group comparison was used for data of unequal variance or non-normal distribution. A $P$ value of less than 0.05 was considered statistically significant.

\section{Results}

PSF $m R N A$ and protein expression in retinal cells. Confluent cultures of RECs, RPCs, and RPEs were evaluated by Northern blot analysis as demonstrated in
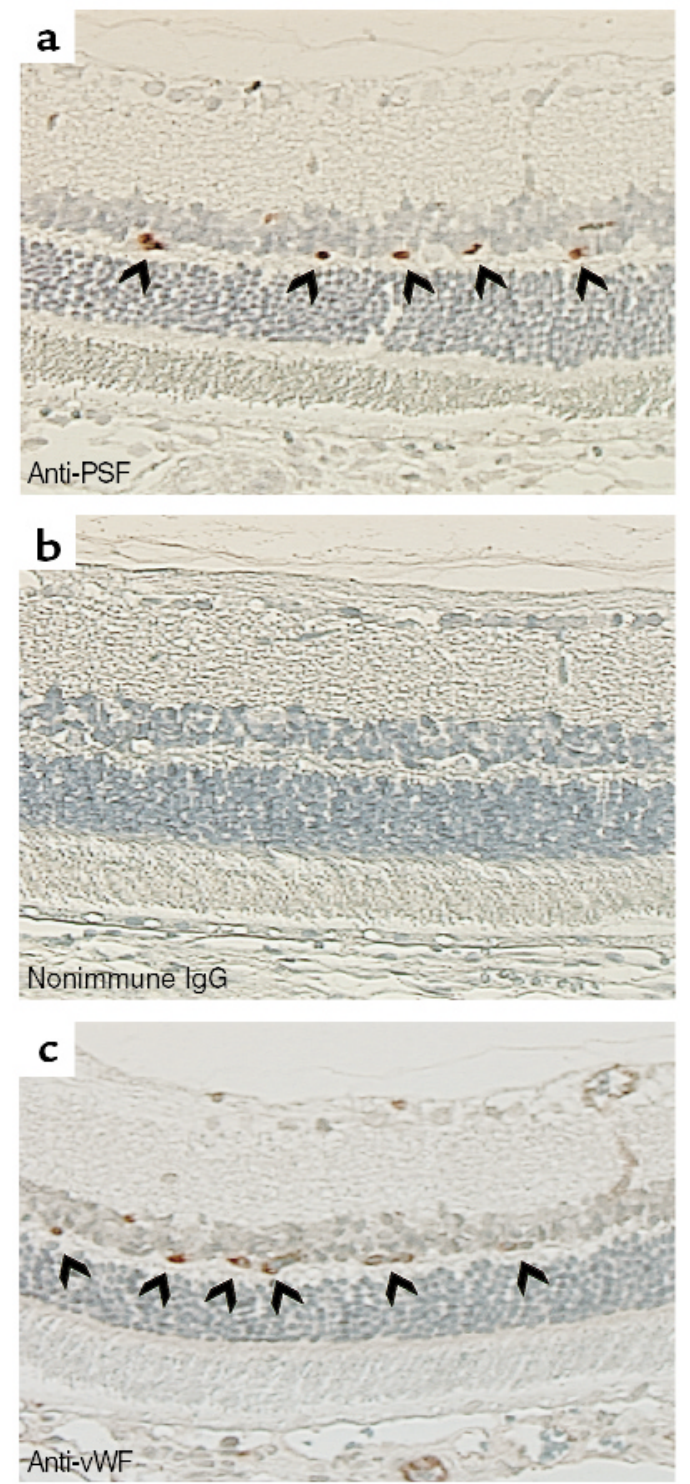

Figure 2

PSF protein is present in normal retina and associated with retinal vessels. Immunohistochemical evaluation of normal rat retina was performed on 3- $\mu$ m-thick paraffin-embedded serial sections using anti-PSF Ab (a), nonimmune $\lg G(\mathbf{b})$, and anti-von Willebrand factor Ab's (c). Immunoreactivity of PSF and von Willebrand factor ( $v W F$ ) exhibited similar distribution (arrowheads). There was no immunoreactivity observed with nonimmune $\operatorname{lgG}$. 


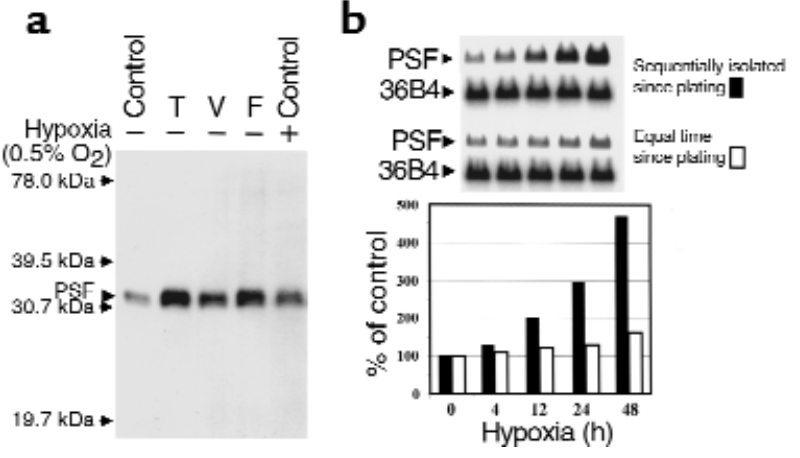

Figure 3

Cytokines and hypoxia associated with worsening of diabetic retinopathy increase PSF expression. (a) Confluent cultures of RECs were exposed to TGF- $\beta$ ( $1 \mathrm{ng} / \mathrm{mL})$, VEGF $(25 \mathrm{ng} / \mathrm{mL})$, bFGF (10 $\mathrm{ng} / \mathrm{mL})$, or hypoxia $\left(0.5 \% \mathrm{O}_{2}, 5 \% \mathrm{CO}_{2}, 94.5 \% \mathrm{~N}_{2}\right)$ for 24 hours before evaluation of the media for PSF protein by Western blot analysis. T, TGF- $\beta$; V, VEGF; F, bFGF. (b) Nearly confluent cultures of RECs were exposed to hypoxic conditions $\left(0.5 \% \mathrm{O}_{2}, 5 \% \mathrm{CO}_{2}, 94.5 \% \mathrm{~N}_{2}\right)$ for the period of time indicated in the figure before isolation of RNA and Northern blot analysis. Cells were either isolated at the same time after initial plating (open bars) or sequentially after plating (filled bars). Representative Northern blot analysis (top) and quantitation (bottom) are presented.

Figure 1. Bovine AECs and aortic SMCs (ASMCs) were studied as nonretinal controls. PSF mRNA was expressed in all cell types. Expression was highest in RPCs, being 4.6-fold greater than in ASMCs $(P<$ $0.001)$ and 3.1- to 6.5-fold greater than in AECs and RECs, respectively $(P=0.001)$.

Immunohistochemistry was performed on $3-\mu \mathrm{m}$ thick paraffin-embedded serial sections of rat retina (Figure 2). PSF protein immunoreactivity was detected primarily in association with retinal vasculature (Figure 2a). Immunostaining with nonimmune IgG was nonreactive (Figure 2b). Immunostaining with anti-von Willebrand factor to identify retinal vessels showed similar localization as with PSF staining (Figure 2c).

PSF mediation by factors implicated in the pathogenesis of diabetic retinopathy. Since advancing diabetic retinopathy is characterized by retinal blood-flow abnormalities, we evaluated whether factors classically associated with worsening retinopathy affected PSF expression. Twenty-four hour exposure of RECs to TGF- $\beta$ (1 $\mathrm{ng} / \mathrm{mL})$, VEGF $(25 \mathrm{ng} / \mathrm{mL})$, bFGF $(10 \mathrm{ng} / \mathrm{mL})$ or hypoxia $\left(0.5 \% \mathrm{O}_{2}, 5 \% \mathrm{CO}_{2}, 94.5 \% \mathrm{~N}_{2}\right)$ increased PSF protein expression 5.3-fold $(P<0.001), 2.6$-fold $(P=$ $0.004), 3.3$-fold $(P=0.003)$, and twofold $(P=0.018)$, respectively (Figure 3a).

Figure $3 \mathrm{~b}$ demonstrates the time course of hypoxia's effect on PSF expression for all cells isolated at the same time after initial plating (open bars) and for all cells placed in hypoxic conditions at the same time, but assayed sequentially thereafter (filled bars). In cells isolated at the same time after initial plating, increases in PSF expression were evident after 12 hours, with increases of $8 \%, 18 \%, 27 \%$, and $58 \%$ after
$4,12,24$, and 48 hours of hypoxia, respectively. For cells assayed sequentially, PSF expression was greater, with increases of $11 \%, 83 \%, 223 \%$, and $373 \%$, respectively. These data suggested that greater cell confluence over the duration of the experiment might result in increased PSF expression.

PSF mRNA expression by RECs was evaluated at $80 \%$ confluency and at $0,1,3$, and 5 days after confluency (Figure 4a). PSF expression increased 6\%, 67\%, 125\%, and $86 \%$ after $0,1,3$, and 5 days of confluency, respectively $(P$ $<0.012$ ). Increased PSF expression correlated with enhanced TGF- $\beta$ mRNA expression in the same cells, which increased $35 \%, 80 \%, 70 \%$, and $70 \%$, respectively.

To assess if this effect was due primarily to cessation of cellular growth, PSF mRNA expression in RECs was assessed after 24-hour exposure to serum starvation (Figure 4b). PSF mRNA expression was inhibited by serum starvation with decreases of $16 \%, 30 \%, 43 \%$, and $81 \%$ in $10 \%$ calf serum (CS), $3 \%$ CS, $1 \%$ CS, and $0.1 \%$ BSA, respectively, as compared with $20 \%$ CS $(P<0.001)$. Decreased PSF expression correlated with reduced TGF- $\beta$ mRNA expression in the same cells, which declined $5 \%, 16 \%, 34 \%$, and $52 \%$, respectively. Thus, the increase in PSF expression observed with confluency did not appear to be mediated primarily by cessation of cellular growth or serum depletion, but perhaps was more closely related to TGF- $\beta$ expression.

TGF- $\beta$ increased REC PSF mRNA expression in a time- and dose-dependent manner (Figure 5, a and b). Statistically significant increases in PSF mRNA expression were initially observed after 4 hours of exposure to $1 \mathrm{ng} / \mathrm{mL}$ TGF- $\beta$ and were still increasing even after 24 hours. PSF mRNA expression was increased $2 \%$, $29 \%, 107 \%$, and $245 \%$ after $1,4,10$, and 24 hours of exposure to TGF- $\beta$, respectively. After 16 hours of exposure, statistically significant increases in PSF a

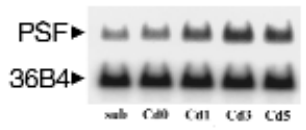

xal rall call ces cas

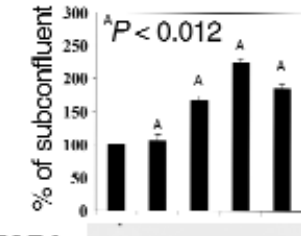

TGF- $\beta+\infty \infty-\infty$ b

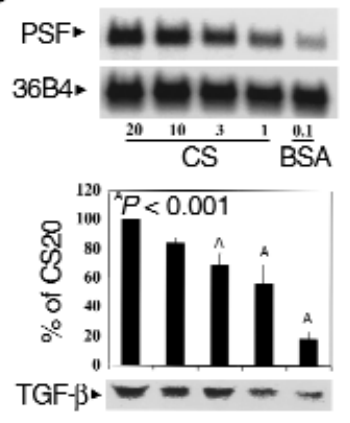

\section{Figure 4}

PSF mRNA expression is increased by cell confluency. (a) REC RNA was isolated at $80 \%$ cell confluency (sub) and at $0,1,3$, and 5 days after confluency (Cd) was achieved. ${ }^{A} P<0.012$. (b) REC RNA was isolated after 24 hours' exposure to media containing $0.1 \%$ BSA or calf serum (CS) at the percentages indicated in the figure. ${ }^{A} P<0.001$. Representative Northern blot analysis for PSF (top), quantitation from multiple experiments after normalization to 36B4 control signal (middle), and representative Northern blot analysis of TGF- $\beta$ RNA expression in the same cells (bottom) are presented. 


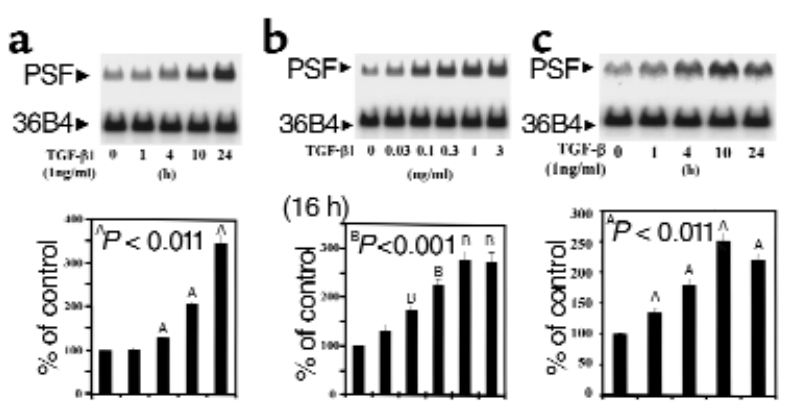

Figure 5

TGF- $\beta$ increases PSF mRNA expression in a time- and dose-dependent manner. RECs were exposed to either $1 \mathrm{ng} / \mathrm{mL}$ TGF- $\beta$ for the times indicated ( $\mathbf{a}$ and $\mathbf{c}$ ) or for 16 hours to the concentrations of TGF- $\beta$ indicated (b). RNA was isolated and Northern blot analysis was performed. Representative Northern blot analysis (top) and quantitation from multiple experiments after normalization to 36B4 control signal (bottom) are presented. ${ }^{A} P<0.011 ;{ }^{B} P<0.001$.

mRNA expression were initially evident at a concentration of $0.03 \mathrm{ng} / \mathrm{mL}$ TGF- $\beta$ and were maximal at 1 $\mathrm{ng} / \mathrm{mL}$. PSF mRNA expression was increased 32\%, $74 \%, 126 \%, 178 \%$, and $174 \%$ by $0.03,0.1,0.3,1.0$, and $3.0 \mathrm{ng} / \mathrm{mL}$ TGF- $\beta$, respectively. In addition, TGF- $\beta$ (1 $\mathrm{ng} / \mathrm{mL}$ ) increased its own expression in RECs by $35 \%$, $80 \%, 152 \%$, and $120 \%$ after 1 , 4, 10, and 24 hours, respectively (Figure 5c; $P<0.011$ ).

Since PKC activation in the retina is an early finding in diabetes associated with decreased RBF, we assessed the effect of PKC activation using PMA in RECs (Figure 6). PMA (100 nM) exposure decreased PSF expression by $35 \%$ and $53 \%$, after 10 and 24 hours, respectively $(P<0.009)$. Figure 7 presents the effect of glucose concentration on PSF protein expression. RECs were cultured for 5 days in 5.5 or $22 \mathrm{mM}$ glucose or $5.5 \mathrm{mM}$ glucose plus $16.5 \mathrm{mM}$ mannitol as an osmotic control. High glucose reduced PSF protein expression 38\% and $23 \%$, compared with normal glucose $(P=0.004)$ or osmotic control conditions $(P=0.023)$.

Retinal PSF expression in vivo is altered by diabetes and correlates with TGF- $\beta$ expression and retinal neovascularization. Since changes in PSF expression could theoretically mediate alterations in RBF (1-3) through its effect on PGI2, we evaluated the expression of PSF and TGF- $\beta$ mRNA in the retina of control and STZ-induced diabetic rats of 2, 4, and 8 weeks' duration. In these animals, $\mathrm{RBF}$ is decreased $38.9 \%(n=24, P=0.007)$ after 2 weeks, $23.9 \%(n=22, P=0.015)$ after 4 weeks $(40)$, and increased $57.6 \%(n=15, P=0.005)$ after 12 weeks of diabetes. As demonstrated in Figure 8a, PSF mRNA expression in diabetic rat retina was $32 \%$ lower than in control animals after 2 weeks of diabetes $(P=0.002)$, not significantly different after 4 weeks of diabetes, and increased $82 \%$ compared with control after 8 weeks of diabetes $(P=0.035)$. This change resulted primarily from a suppression of the gradual decline in retinal PSF expression present in control animals over the 8week period $(P=0.003)$.
To determine whether TGF- $\beta$ expression might be mediating this effect, we evaluated retinal TGF- $\beta$ mRNA expression in the same animals (Figure $8 \mathrm{~b}$ ). Although TGF- $\beta$ expression tended to be higher in diabetic animals with increasing duration of diabetes, this change was not statistically significant until after 8 weeks of diabetes. After 8 weeks of diabetes, retinal TGF- $\beta$ expression was $56 \%$ higher in diabetic than in nondiabetic animals $(P<0.001)$. TGF- $\beta$ expression in nondiabetic animals did not change significantly over the 8-week period. Since VEGF can increase PSF expression (Figure 3), we evaluated retinal VEGF expression in these same animals. Although VEGF expression in diabetic retinas tended to increase over time, this finding was not statistically significant (data not shown).

To determine if retinal PSF mRNA expression might correlate with proliferative retinopathy where RBF is substantially increased $(2,41,42)$, a well-described murine model of oxygen-induced retinal proliferation was employed (28). In this model, retinas become hypoxic at postnatal day 12 (P12), and retinal neovascularization developed in $100 \%$ of animals over P13-P17. Neovascularization regresses gradually after P20. Figure 9 demonstrates retinal PSF mRNA expression in these animals and their littermate controls. All lanes were loaded equally, as assessed by $18 \mathrm{~S}$ rRNA signal. PSF expression increased during the period of neovascularization. PSF expression was maximal at P17, the same time when maximal retinal neovascularization is observed. Slight increases in PSF expression appeared within 6 hours of when retinas became hypoxic. PSF expression returned to control levels by P25, when neovascular regression is well established. Hyperoxia did not alter PSF mRNA expression significantly (P7-6H, P8H, and P12-0H lanes in Figure 9a).

Evaluation of PSF bioactivity. PSF stimulates production of PGI2, which is rapidly converted to the stable metabolite 6-keto-PGF1 $\alpha$. To determine if retinal cells synthesized PGI2, the concentration of 6-keto-PGF1 $\alpha$ was measured in media conditioned for 24 hours by RECs and RPCs (Figure 10a). Media conditioned in the

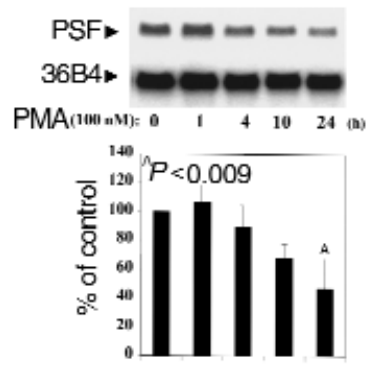

\section{Figure 6}

PMA decreases PSF mRNA expression in RECs. RECs were exposed to $100 \mathrm{nM}$ PMA for the times indicated in the figure, after which RNA was isolated and Northern blot analysis performed. Representative Northern blot analysis (top) and quantitation from multiple experiments after normalization to $36 \mathrm{~B} 4$ control signal (bottom) are presented. ${ }^{A} P<0.009$ 


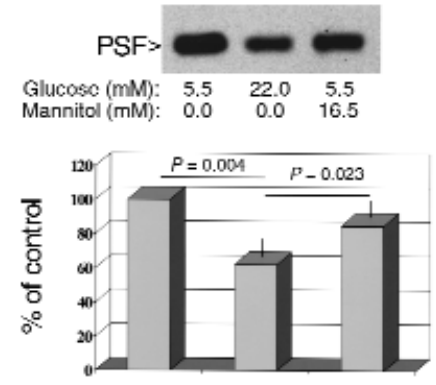

\section{Figure 7}

High glucose concentrations reduce PSF protein expression. RECs were cultured for 5 days in media containing glucose or mannitol (osmotic control) as indicated. Representative Western blot analysis is presented (top) as is quantitation from three experiments (bottom).

absence of cells did not contain detectable concentrations of 6-keto-PGF1 $\alpha$, whereas media conditioned by RPCs and RECs for 24 hours contained significantly elevated 6-keto-PGF1 $\alpha$ concentrations $(P<0.001)$. Bioactive recombinant PSF protein does not yet exist, and purified PSF protein is not available in sufficient quantity to evaluate. Thus, to determine if this PGI2 synthesis was mediated by PSF, we used an antisense phosphorothioate oligodeoxynucleotide (ODN). Transfection of RPCs with the antisense PSF ODN reduced PSF protein expression by $72 \%(P<0.001)$, an effect not observed using either sense or randomized ODNs as controls (Figure 10b).

The specific ability of RPC-secreted PSF to induced PGI2 expression by RECs was evaluated by transfecting RPCs with the PSF antisense or sense ODN before using them to condition media (Figure 10c). Conditioned media were concentrated through a $10,000-$ MW cutoff centrifugation filter that retains PSF (31 $\mathrm{kDa}$ ), but not PIG2 or the ODN. RECs were then exposed for 1 hour to the RPC-conditioned media, and PGF1 $\alpha$ accumulation in the media was measured. Media alone (media) contained little PGI2. RPC-conditioned media that was subjected to the same collection and concentration procedures as the remaining experiments, but that was not exposed to RECs (ctl), had the same low level of PGI2, demonstrating that essentially all RPC-produced PGI2 was removed by the concentration process. Media that was conditioned in the absence of RPCs and then added to RECs (nonconditioned media $[\mathrm{NCM}]$ ) showed a 4.5 -fold accumulation of PGI2 $(P<0.001)$, consistent with basal REC-PGI2 synthesis. Media conditioned by RPCs in the presence of PSF sense (S) or randomized (R) ODN further increased REC PGF1 $\alpha$ production by more than fourfold $(P<0.003)$, consistent with PSF-like activity in the RPC-conditioned media. However, media conditioned in the presence of PSF antisense ODN-treated (AS-treated) RPCs resulted in REC-PGI2 expression that was $75 \%$ less than with randomized ODN $(P=0.002), 71 \%$ less than sense ODN $(P=0.006)$, and was only marginally increased $(30 \%, P=0.062)$ as compared with NCM. These data suggest that PSF in RPC-conditioned media mediates the majority of its PGI2-stimulating activity in REC.

PSF alters retinal hemodynamics in vivo. PGI2 is vasodilatory in nonocular tissues in vivo (43) and might therefore increase retinal blood flow. PGI2 $\left(10^{-6} \mathrm{M}\right.$ final) was injected intravitreally and $\mathrm{MCT}$ and $\mathrm{RBF}$ were measured using video fluorescein angiography in nondiabetic and diabetic rats (Figure 11). After 20 minutes, the time at which a maximal retinal response was noted, PGI2 decreased MCT and increased RBF by $27 \%$ and $53 \%$ in control animals $(P<0.021)$ and $22 \%$ and $42 \%$ in diabetic animals $(P<0.07)$, respectively. MCT and RBF returned to base line within 40 minutes. Injection of vehicle alone did not alter MCT or RBF. PGI2 injection was associated with a tendency for dilation of the major arteries (5.9-6.1 pixels, $P=0.22$ ) and a more marked dilation of the major veins, especially in diabetic animals (control: 7.2-7.5 pixels, $P=0.06$; diabetic: 6.8-7.3 pixels, $P=0.027$ ).

To determine whether PSF secreted by retinal cells could induce changes in retinal hemodynamics, media were conditioned for 24 hours by RPCs, either in the presence of PSF sense or antisense ODN. Media were concentrated 25 -fold, and $10 \mu \mathrm{L}$ was injected intravitreally in the rat (2.1-fold effective final concentration). MCT and $\mathrm{RBF}$ were measured after the times indicated in Figure 12. Media conditioned in the presence of PSF antisense ODN resulted in increases in control animals of $56 \%(P=$ $0.012), 114 \%(P<0.001)$, and $53 \%(P=0.022)$ in MCT after 30,40 , and 50 minutes, respectively, as compared with media conditioned with sense control (Figure 12a). In diabetic animals MCT increased $86 \%(P=0.002)$ and $77 \%$ $(P=0.023)$ after 30 and 45 minutes, respectively. Media conditioned with PSF sense ODN did not significantly decrease MCT, except in diabetic animals at 30 minutes (25\%, $P=0.037)$. Concomitantly, RBF was decreased $40 \%$ $(P=0.009), 54 \%(P<0.001)$, and $37 \%(P=0.027)$ as compared with sense control after 30,40, and 50 minutes in control animals, respectively (Figure 12b). In diabetic ani-

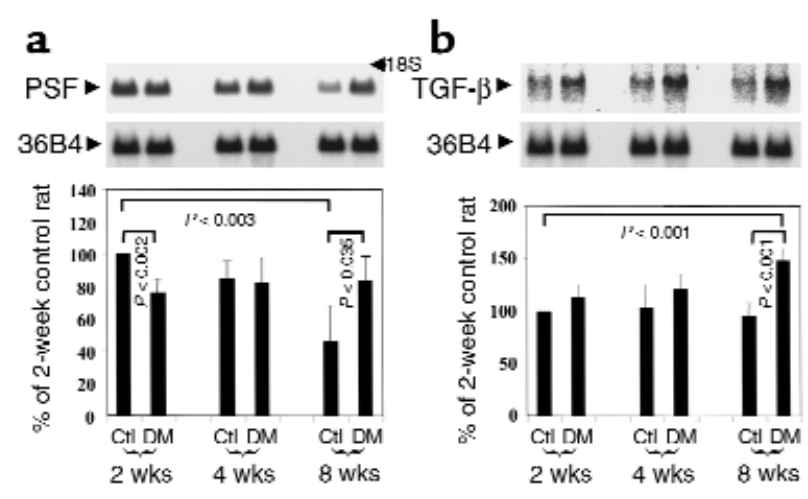

Figure 8

Duration of diabetes regulates retinal PSF and TGF- $\beta$ expression. Retinas were isolated from Sprague-Dawley rats after 2, 4, and 8 weeks of STZ-induced diabetes. RNA was isolated and Northern blot analysis for PSF (a) and TGF- $\beta$ (b) performed. Representative Northern blot analysis (top) and quantitation from multiple experiments after normalization to 36B4 control signal (bottom) are presented. 


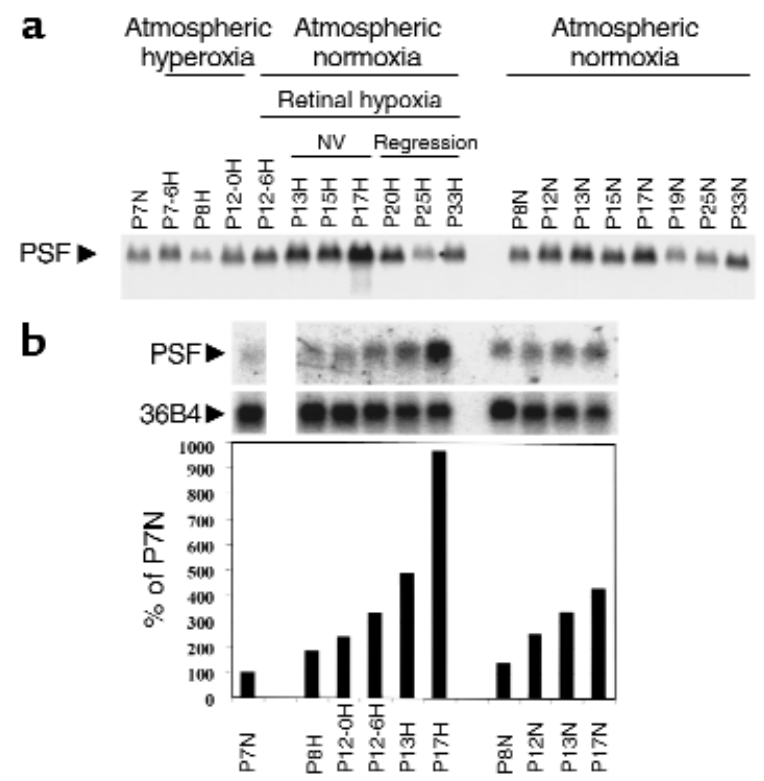

Figure 9

PSF mRNA expression increases during retinal neovascularization. The oxygen-induced model of retinopathy in the neonatal mouse was used. The atmospheric conditions at each stage, as well as the periods of retinal hypoxia and retinal neovascularization and regression, are indicated. Representative Northern blot analysis is presented for two different sets of experiments. A time course including the entire retinal response period (a) and an experiment focusing on the period of maximal retinal neovascularization (b) are presented. Quantitation of the experiment in $\mathbf{b}$ is also presented. All lanes demonstrate equal 18S RNA loading. As described previously, 36B4 undergoes some developmental regulation in this mouse model $(58,59)$. PX, postnatal day $\mathrm{X} ; \mathrm{N}$, normoxic; $\mathrm{H}$, hypoxic. When present, the number after the dash indicates hours after change in atmospheric condition.

mals RBF was reduced $52 \%(P<0.001)$ and $47 \%(P=$ $0.013)$ after 30 and 45 minutes, respectively. Media conditioned with PSF sense ODN tended to increase RBF, but this was only significant at 40 minutes $(25 \%, P=$ $0.037)$ in control animals and in diabetic animals (30 minutes: $41 \%, P=0.045$; 45 minutes: $21 \%, P=0.045$ ). PSF antisense-conditioned media resulted in increases of $60 \%$ $(P=0.036), 99 \%(P<0.001)$, and $73 \%(P=0.032)$ in MCT and decreases of $48 \%(P=0.044), 55 \%(P=0.021)$, and $47 \%$ $(P=0.070)$ in RBF after 30,40 , and 50 minutes, respectively, as compared with base line (0 minutes). Diabetic animals showed a similar tendency with a statistically significant RBF reduction of $19 \%$ at 30 minutes $(P=0.029)$. Both arterial and venous diameters were increased by media conditioned in the presence of PSF sense control and decreased by media conditioned with PSF antisense ODN at all time points, although these changes reached statistical significance only for arterial diameters in diabetic animals ( 30 minutes: $6.0-5.6$ pixels, $P<0.001 ; 45$ minutes: $6.0-5.7$ pixels, $P=0.013$ ).

\section{Discussion}

PSF is a recently purified protein that acts on vascular endothelial cells to stimulate the synthesis of prostacyclin (PGI2) (2). However, little is known regarding the role of PSF in the diabetic and normal retina. Our data strongly suggest that PSF may play a significant role in mediating diabetes-associated hemodynamic alterations in the retina.

The presence of PSF in the retina is supported by several findings in this study. Immunoreactive PSF is detected in the in vivo retina, apparently primarily localized to the vasculature. PSF mRNA is readily detectable from total retina both in rats and neonatal mice. PSF mRNA and protein are expressed in numerous ocular cell types in vitro at levels comparable to those observed in AECs and SMCs (4, 5, 7-11). We demonstrate that the constitutive expression in retinal microvascular pericytes is greater than threefold high-

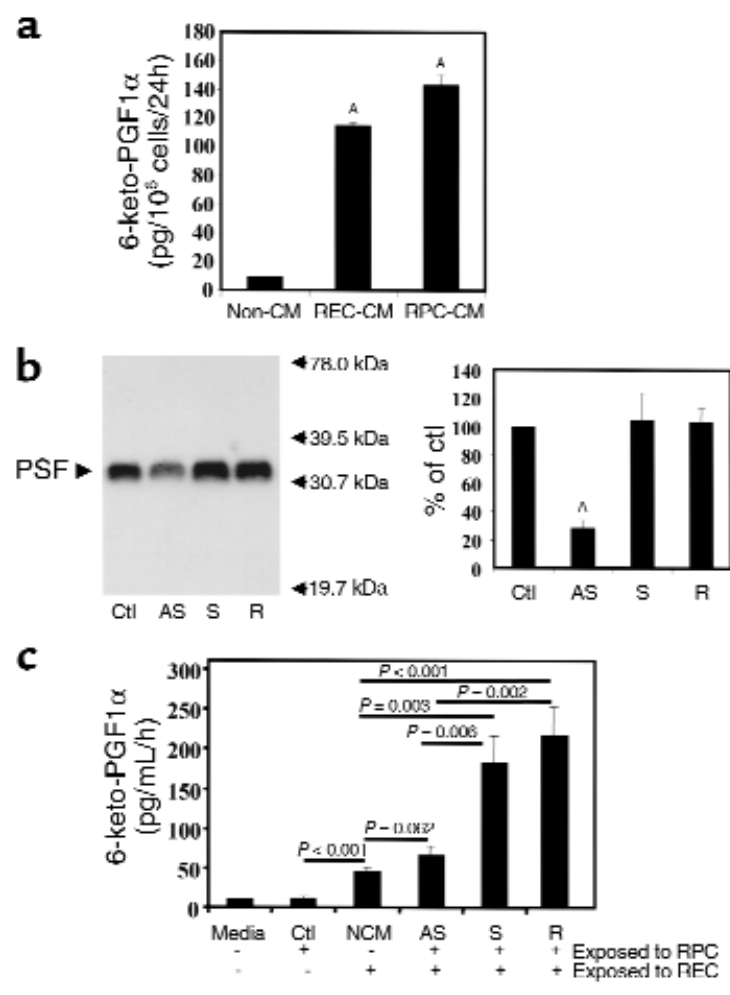

Figure 10

Stimulation of REC PGF1 $\alpha$ production by RPC-conditioned media is primarily mediated by PSF. PGF1 $\alpha$ synthesis by REC and RPC is shown in (a). Media was either conditioned in the absence of cells (non-CM), or conditioned by REC (REC-CM), or RPC (RPC-CM) for 24 hours before being assayed for PGF1 $\alpha$. (b) The effect of transfecting PSF antisense ODN (AS), sense ODN control (S), random ODN control $(\mathrm{R})$, or lipofectamine only ( $\mathrm{ct}$ ) before evaluation of PSF protein by Western blot analysis after 24 hours. A representative Western blot (left) and quantitation of multiple experiments (right) are shown. (c) The ability of PSF in RPC-conditioned media to increase REC PGI2 production. RPCs were transfected with PSF antisense (AS), sense (S), or random (R) ODN before using them to condition media. RPC-conditioned media were concentrated through a 10,000-MW cutoff centrifugation filter that retains PSF but not PIG2 or the ODN. RECs were then exposed for 1 hour to the RPC-conditioned media and PGF1 $\alpha$ accumulation in the media measured. Basal media alone (media), media conditioned by RPCs but not exposed to RECs (ctl), and RECs exposed to media conditioned in the absence of RPCs (NCM) were evaluated as controls. ${ }^{A} P<0.001$. 

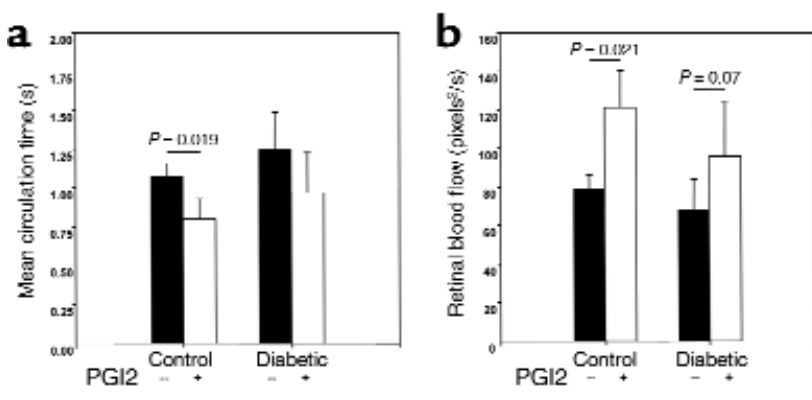

Figure 11

PGI2 increases RBF in vivo. PGI2 dissolved in $10 \mu \mathrm{L}$ of $2.5 \%$ Emulphor was injected intravitreally into rat eyes for a final concentration of $10^{-6}$ M. MCT (a) and RBF (b) were measured after 20 minutes by fluorescein dye-dilution technique. Injection of vehicle alone had no effect. Results are expressed as mean $\pm S D ; n=5$ animals per group.

er than in the other retinal cells tested. Pericyte loss is a characteristic early finding in diabetic retinopathy (44-46), suggesting that pericyte loss might reduce PSF, resulting in decreased PGI2, relative vasoconstriction, and decreased RBF as characteristically observed with early diabetes in humans $(1,2)$ and rats (30-32). However, this effect would be dependent upon the time course of pericyte loss and, since RECs also express PSF, the relative autocrine and paracrine contribution cannot be fully determined from these studies.

The bioactivity of PSF expressed by retinal cells is supported by several findings. RPC-conditioned media increase PGI2 expression by RECs. Furthermore, induction of PGI2 expression in RECs by RPC-conditioned media is inhibited by treatment of RPCs with a PSF-specific antisense phosporothioate ODN that effectively suppresses PSF protein expression. In contrast, PGI2 expression is not inhibited by sense or randomized ODNs that have no effect on PSF protein expression. We used the antisense approach since bioactive recombinant PSF is not currently available to test directly its action on retinal cells. Conditioned media contains many possible bioactive factors. The difference observed between antisense and sense/randomized ODN-conditioned media theoretically should reflect only the effect of inhibiting the expression of PSF; however, a possible effect of the antisense ODN on factors other than PSF that subsequently mediate the response cannot be completely ruled out by this approach.

PSF bioactivity is also suggested by the in vivo retinal response to retinal cell-conditioned media. As expected, direct stimulation of the retina with PGI2 induced vasodilation, decreased MCT, and increased RBF. Similarly, retinal cell-conditioned media without PSF inhibition tended to decrease MCT and increase RBF while increasing vasodilation. This was especially evident in diabetic animals where statistical significance was observed for arterial diameters, MCT, and RBF. The relatively small magnitude of this effect in nondiabetic animals could result from either near-maximal vasodilatory activity in vivo at base line or from inhibitory/vasoconstrictive factors counteracting PSF activity in the conditioned media. In addition, the video fluorescein angiography technique does not detect small-diameter changes in major retinal vessels, nor does it evaluate smaller-caliber retinal vessels that are thought to mediate the majority of blood flow control. In contrast, media conditioned with PSF antisense ODN resulted in a marked increase in MCT, decrease in RBF, and a tendency for vascular constriction. These data suggest that PSF might be counteracting inhibitory/vasoconstrictive factors in the conditioned media which, when PSF expression is inhibited, are free to act unopposed. As discussed earlier, however, there are caveats with regard to the use of conditioned media in the absence of more specific agonists and antagonists.

The retinal hemodynamic effects of PSF are probably mediated in part through the vasodilatory effects of PGI2 (19;20). Increased PSF activity would be expected to increase PGI2, induce vasodilation, decrease MCT, and increase RBF. In all experiments, both arterial and venous vessel-diameter changes were consistent with expected results. In diabetic animals, the arterial changes were marked $(P<0.001)$. However, in nondiabetic animals the magnitude of the change was small. The limitations in measuring small-diameter changes in the major retinal vessels using the dye-dilution technique has been discussed in detail in previous publications (31). In addition, large-diameter changes in major retinal vessels are not required because the microvasculature is the primary site of RBF regulation and quantitative measurements from these vessels are not possible with the current resolution capabilities of this technique.

\section{a}
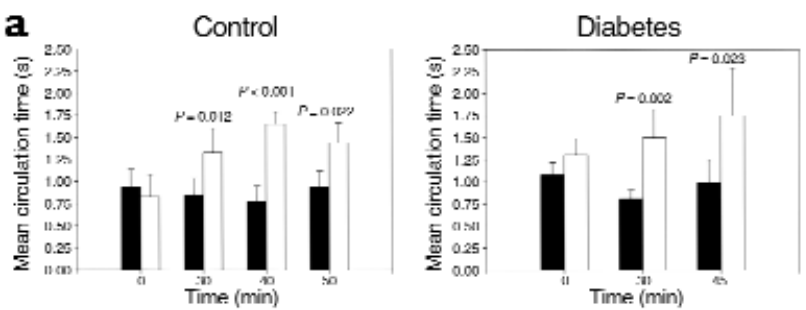

b
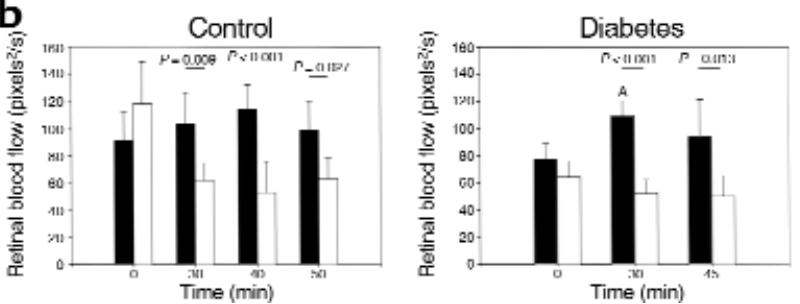

\section{Figure 12}

PSF secreted by RPCs increases RBF in vivo. Media was conditioned for 24 hours by RPCs in the presence of PSF sense (filled bars) or antisense (open bars) phosphorothioate ODN. Media was then concentrated 25 -fold and $10 \mu \mathrm{L}$ was injected intravitreally in the rat, resulting in a final media concentration of approximately 2.5 -fold. $\operatorname{MCT}(\mathbf{a})$ and RBF (b) were measured by fluorescein dye-dilution technique at the indicated times. $n=5$ animals per group. 
Several additional findings suggest that PSF may play a significant role in mediating retinal vascular function in diabetes. In vitro PSF protein expression was increased in retinal cells by factors such as TGF- $\beta$, VEGF, bFGF, and hypoxia. The association of these factors with advancing diabetic retinopathy has been well established $(29,47-52)$. Increasing cellular confluency also resulted in significantly increased PSF expression, possibly mediated, at least in part, by TGF$\beta$. This suggests that maturation of neovascularization characteristic of advanced retinopathy might increase PSF expression and partially account for increases in RBF observed at this stage of retinopathy. This hypothesis is consistent with the time course of PSF mRNA expression observed in the neonatal mouse retina where PSF expression peaked as retinal neovascularization became maximal (P17).

Further association of PSF and retinal hemodynamics is observed in the rat retina where PSF mRNA expression is higher in animals with 8 weeks of diabetes than in age-matched nondiabetic controls. Conversely, after 2 weeks of diabetes, diabetic animals express less PSF mRNA than do their nondiabetic controls. Both of these findings are consistent with the changes in RBF characteristic of the diabetic animals at these stages (32). Interestingly, this change primarily appears to be the result of a developmental reduction in PSF expression in nondiabetic rats that is not evident in the diabetic rats. It is unknown if this effect is mediated by secondary factors, such as weight gain. PSF expression may remain elevated in diabetic rats due to an increase in retinal TGF- $\beta$ expression observed in the retina after 8 weeks of diabetes. TGF- $\beta$ expression and activity is increased in diabetes and by hypoxia (49). We also observed that TGF- $\beta$ can further enhance its own expression. However, a causal relationship with TGF- $\beta$ is not proven by these experiments. Retinal VEGF expression only increased minimally over the short course of these experiments, suggesting that VEGF is not a primary mediator of increased PSF expression in these early stages of retinopathy.

Overall, our data suggest that PSF may play an important role in mediating the changes in retinal hemodynamics characteristic of diabetic retinopathy. Diabetes in its early stages is associated with elevated serum glucose concentration, loss of retinal pericytes, and an increase in PKC activity, all of which may result in reduced PSF, decreased PGI2 production, increased resistance to flow associated with relative vasoconstriction, and decreased RBF. Retinal PKC/DAG activity is increased in the rat retina within 1-2 weeks of diabetes and maintained for several years $(30 ; 30 ; 35 ; 53)$. Thus, the contribution of PKC may predominate early in diabetes, whereas other factors may account for later changes. RPCs have been reported to induce significant prostacyclin production in RECs (54), and prostacyclin is the major prostaglandin synthesized by RPCs in culture (55). Pericyte loss would reduce PGI2 concentrations due to declines in both PSF and PGI2 contributed by the per- icytes themselves. However, with advancing diabetic retinopathy, TGF- $\beta$ and retinal hypoxia increase. Retinal hypoxia leads to VEGF expression, TGF- $\beta$ activation, and bFGF release, which further increases VEGF expression. All these factors increase PSF expression and PGI2 production, possibly leading to the vasodilation and increased RBF characteristic of advanced diabetic retinopathy. With maturation of the retinal neovascularization, further increases in RBF may be mediated by PSF expression in endothelial cells and recovering pericytes within these maturing vessels $(56,57)$.

In summary, we observe that PSF is localized to the retinal vasculature in vivo and is expressed by numerous cell types including RECs and RPCs. PSF increases PGI2 production in RECs, which is vasodilatory and results in increased retinal blood flow in vivo. Retinal PSF expression is reduced during early diabetes and increased in more advanced diabetes compared with nondiabetic retinas. Furthermore, we demonstrate that PSF is tightly regulated by various factors associated with diabetic retinopathy, with decreased expression induced by factors predominating in early retinopathy, and increased expression associated with factors involved in advancing retinopathy. These results imply that PSF may serve an important role in the diabetic retina and suggest a mechanism to account for the biphasic RBF abnormalities classically associated with progressing diabetic retinopathy.

\section{Acknowledgments}

This work was supported in part by NIH grant EY10827 (to L.P. Aiello). The Joslin Diabetes Center is the recipient of NIH Diabetes and Endocrinology Research Center grant 36836. The authors thank Jerry Cavallerano, Susan Rook, and Alex Vogel for their assistance with this project.

1. Bursell, S.E., et al. 1996. Retinal blood flow changes in patients with insulin-dependent diabetes mellitus and no diabetic retinopathy. Am.J. Physiol. 270:R61-R70.

2. Clermont, A.C., Aiello, L.P., Mori, F., Aiello, L.M., and Bursell, S.E. 1997. Vascular endothelial growth factor and severity of nonproliferative diabetic retinopathy mediate retinal hemodynamics in vivo: a potential role for vascular endothelial growth factor in the progression of nonproliferative diabetic retinopathy. Am. J. Ophthalmol. 124:433-446.

3. Konno, S., et al. 1996. Retinal blood flow changes in type I diabetes. A long-term follow-up study. Invest. Ophthalmol. Vis. Sci. 37:1140-1148.

4. Yamauchi, T., et al. 1994. Purification and molecular cloning of prostacyclin-stimulating factor from serum-free conditioned medium of human diploid fibroblast cells. Biochem. J. 303:591-598.

5. Mizushima, S., et al. 1996. Isolation and characterization of the human chromosomal gene for prostacyclin-stimulating factor. J. Biochem. (Tokyo). 120:929-933.

6. Ono, Y., et al. 1998. Reduced expression of a novel peptide, prostacyclinstimulating factor, in the kidneys of streptozotocin-induced diabetic rats. J. Diabetes Complications. 12:252-258.

7. Sekiguchi, N., et al. 1997. Immunohistochemical study of prostacyclinstimulating factor (PSF) in the diabetic and atherosclerotic human coronary artery. Diabetes. 46:1627-1632.

8. Umeda, F., et al. 1996. Prostacyclin-stimulating factor, novel protein, and diabetic angiopathy. Diabetes. 45(Suppl. 3):S111-S113.

9. Ono, Y., et al. 1998. Effect of high glucose concentrations on prostacyclin-stimulating factor mRNA expression in cultured aortic smooth muscle cells. Diabetologia. 41:134-140.

10. Masakado, M., et al. 1995. Immunohistochemical localization of a novel peptide, prostacyclin-stimulating factor (PSF), in human tissues. Thromb. Haemost. 74:1407-1410.

11. Ono, Y., et al. 1994. Expression of prostacyclin-stimulating factor, a 
novel protein, in tissues of Wistar rats and in cultured cells. Biochem. Biophys. Res. Commun. 202:1490-1496.

12. Umeda, F., et al. 1998. Increased mRNA expression of a novel prostacyclinstimulating factor in human colon cancer. J. Gastroenterol. 33:213-217.

13. Weksler, B.B., Ley, C.W., and Jaffe, E.A. 1978. Stimulation of endothelial cell prostacyclin production by thrombin, trypsin, and the ionophore A 23187. J. Clin. Invest. 62:923-930.

14. Coughlin, S.R., Moskowitz, M.A., Zetter, B.R., Antoniades, H.N., and Levine, L. 1980. Platelet-dependent stimulation of prostacyclin synthesis by platelet-derived growth factor. Nature. 288:600-602.

15. Rossi, V., Breviario, F., Ghezzi, P., Dejana, E., and Mantovani, A. 1985. Prostacyclin synthesis induced in vascular cells by interleukin-1. Science. 229:174-176.

16. Kawakami, M., et al. 1986. Cachectin/TNF as well as interleukin-1 induces prostacyclin synthesis in cultured vascular endothelial cells. Biochem. Biophys. Res. Commun. 141:482-487.

17. Ristimaki, A., Ylikorkala, O., Perheentupa, J., and Viinikka, L. 1988. Epidermal growth factor stimulates prostacyclin production by cultured human vascular endothelial cells. Thromb. Haemost. 59:248-250.

18. Ristimaki, A. 1989. Transforming growth factor alpha stimulates prostacyclin production by cultured human vascular endothelial cells more potently than epidermal growth factor. Biochem. Biophys. Res. Commun. 160:1100-1105.

19. Hardy, P., et al. 1998. A major role for prostacyclin in nitric oxideinduced ocular vasorelaxation in the piglet. Circ. Res. 83:721-729.

20. Dodge, A.B., Hechtman, H.B., and Shepro, D. 1991. Microvascular endothelial-derived autacoids regulate pericyte contractility. Cell Motil. Cytoskeleton. 18:180-188.

21. Murohara, T., et al. 1998. Vascular endothelial growth factor/vascular permeability factor enhances vascular permeability via nitric oxide and prostacyclin. Circulation. 97:99-107.

22. Wheeler-Jones, C., et al. 1997. Vascular endothelial growth factor stimulates prostacyclin production and activation of cytosolic phospholipase $\mathrm{A} 2$ in endothelial cells via $\mathrm{p} 42 / \mathrm{p} 44$ mitogen-activated protein kinase. FEBS Lett. 420:28-32.

23. Rymaszewski, Z., et al. 1992. Human retinal vascular cells differ from umbilical cells in synthetic functions and their response to glucose. Proc. Soc. Exp. Biol. Med. 199:183-191.

24. Hata, Y., Duh, E., Zhang, K., Robinson, G.S., and Aiello, L.P. 1998. Transcription factors $\mathrm{Sp} 1$ and $\mathrm{Sp} 3$ alter vascular endothelial growth factor receptor expression through a novel recognition sequence. J. Biol. Chem. 273:19294-19303.

25. Chomczynski, P., and Sacchi, N. 1987. Single-step method of RNA isolation by acid guanidinium thiocyanate-phenol-chloroform extraction. Anal. Biochem. 162:156-159.

26. Sharma, K., and Ziyadeh, F.N. 1994. Renal hypertrophy is associated with upregulation of TGF-beta 1 gene expression in diabetic BB rat and NOD mouse. Am. J. Physiol. 267:F1094-F1101.

27. Aiello, L.P., Robinson, G.S., Lin, Y.W., Nishio, Y., and King, G.L. 1994. Identification of multiple genes in bovine retinal pericytes altered by exposure to elevated levels of glucose by using mRNA differential display. Proc. Natl. Acad. Sci. USA. 91:6231-6235.

28. Smith, L.E., et al. 1994. Oxygen-induced retinopathy in the mouse. Invest. Ophthalmol. Vis. Sci. 35:101-111

29. Aiello, L.P., et al. 1995. Suppression of retinal neovascularization in vivo by inhibition of vascular endothelial growth factor (VEGF) using soluble VEGF-receptor chimeric proteins. Proc. Natl. Acad. Sci. USA. 92:10457-10461.

30. Shiba, T., et al. 1993. Correlation of diacylglycerol level and protein kinase $\mathrm{C}$ activity in rat retina to retinal circulation. Am. J. Physiol. 265:E783-E793.

31. Takagi, C., et al. 1995. Reversal of abnormal retinal hemodynamics in diabetic rats by acarbose, an alpha-glucosidase inhibitor. Proc. Natl. Acad. Sci. USA. 92:10457-10461

32. Clermont, A.C., et al. 1994. Normalization of retinal blood flow in diabetic rats with primary intervention using insulin pumps. Invest. Ophthalmol. Vis. Sci. 35:981-990.

33. Takagi, C., et al. 1996. Endothelin-1 action via endothelin receptors is a primary mechanism modulating retinal circulatory response to hyperoxia. Invest. Ophthalmol. Vis. Sci. 37:2099-2109.

34. Takagi, C., et al. 1996. Regulation of retinal hemodynamics in diabetic rats by increased expression and action of endothelin-1. Invest. Ophthalmol. Vis. Sci. 37:2504-2518.
35. Ishii, H., et al. 1996. Amelioration of vascular dysfunctions in diabetic rats by an oral PKC beta inhibitor. Science. 272:728-731.

36. Koyama, T., et al. 1990. Retinal circulation times in quantitative fluorescein angiography. Graefes. Arch. Clin. Exp. Ophthalmol. 228:442-446.

37. Riva, C.E., Feke, G.T., and Ben-Sira, I. 1978. Fluorescein dye-dilution technique and retinal circulation. Am. J. Physiol. 234:H315-H322.

38. Bulpitt, C.J., and Dollery, C.T. 1971. Estimation of retinal blood flow by measurement of the mean circulation time. Cardiovasc. Res. 5:406-412.

39. Aiello, L.P., et al. 1997. Vascular endothelial growth factor-induced retinal permeability is mediated by protein kinase $C$ in vivo and suppressed by an orally effective beta-isoform-selective inhibitor. Diabetes. 46:1473-1480.

40. Higashi, S., Clermont, A.C., Dhir, V., and Bursell, S.E. 1998. Reversibility of retinal flow abnormalities is disease-duration dependent in diabetic rats. Diabetes. 47:653-659.

41. Grunwald, J.E., Riva, C.E., Sinclair, S.H., Brucker, A.J., and Petrig, B.L. 1986. Laser Doppler velocimetry study of retinal circulation in diabetes mellitus. Arch. Ophthalmol. 104:991-996.

42. Yoshida, A., et al. 1983. Retinal blood flow alterations during progression of diabetic retinopathy. Arch. Ophthalmol. 101:225-227.

43. Barst, R.J., Maislin, G., and Fishman, A.P. 1999. Vasodilator therapy for primary pulmonary hypertension in children. Circulation. 99:1197-1208.

44. Cogan, D.G., and Kuwabara, T. 1963. Capillary shunts in the pathogenesis of diabetic retinopathy. Diabetes. 12:293-300.

45. Cogan, D.G., Toussaint, D., and Kuwabara, T. 1961. Retinal vascular patterns. IV. Diabetic retinopathy. Arch. Ophthalmol. 66:366-378.

46. Toussaint, D., Cogan, D.G., and Kuwabara, T. 1999. Extravascular lesions of diabetic retinopathy. Arch. Ophthalmol. 67:42-47.

47. Aiello, L.P., et al. 1994. Vascular endothelial growth factor in ocular fluid of patients with diabetic retinopathy and other retinal disorders. N. Engl. J. Med. 331:1480-1487.

48. Adamis, A.P., et al. 1994. Increased vascular endothelial growth factor levels in the vitreous of eyes with proliferative diabetic retinopathy. Am. J. Ophthalmol. 118:445-450.

49. Behzadian, M.A., Wang, X.L., Shabrawey, M., and Caldwell, R.B. 1998. Effects of hypoxia on glial cell expression of angiogenesis-regulating factors VEGF and TGF-beta. Glia. 24:216-225.

50. Hueber, A., Wiedemann, P., Esser, P., and Heimann, K. 1996. Basic fibroblast growth factor mRNA, bFGF peptide and FGF receptor in epiretinal membranes of intraocular proliferative disorders (PVR and PDR). Int. Ophthalmol. 20:345-350.

51. Seghezzi, G., et al. 1998. Fibroblast growth factor-2 (FGF-2) induces vascular endothelial growth factor (VEGF) expression in the endothelial cells of forming capillaries: an autocrine mechanism contributing to angiogenesis. J. Cell Biol. 141:1659-1673.

52. Hata, Y., Rook, S.L., and Aiello, L.P. 1999. Basic fibroblast growth factor induces expression of VEGF receptor KDR through a protein kinase $\mathrm{C}$ and $\mathrm{p} 44 / \mathrm{p} 42$ mitogen-activated protein kinase-dependent pathway. Diabetes. 48:1145-1155.

53. Xia, P., et al. 1994. Characterization of the mechanism for the chronic activation of diacylglycerol-protein kinase $\mathrm{C}$ pathway in diabetes and hypergalactosemia. Diabetes. 43:1122-1129.

54. Yamagishi, S., Kobayashi, K., and Yamamoto, H. 1993. Vascular pericytes not only regulate growth, but also preserve prostacyclin-producing ability and protect against lipid peroxide-induced injury of co-cultured endothelial cells. Biochem. Biophys. Res. Commun. 190:418-425.

55. Hudes, G.R., Li, W.Y., Rockey, J.H., and White, P. 1988. Prostacyclin is the major prostaglandin synthesized by bovine retinal capillary pericytes in culture. Invest. Ophthalmol. Vis. Sci. 29:1511-1516.

56. Sato, Y., and Rifkin, D.B. 1989. Inhibition of endothelial cell movement by pericytes and smooth muscle cells: activation of a latent transforming growth factor-beta 1-like molecule by plasmin during co-culture. $J$. Cell Biol. 109:309-315.

57. Ishibashi, T., Inomata, H., Sakamoto, T., and Ryan, S.J. 1995. Pericytes of newly formed vessels in experimental subretinal neovascularization. Arch. Ophthalmol. 113:227-231.

58. Pierce, E.A., Avery, R.L., Foley, E.D., Aiello, L.P., and Smith, L.E. 1995. Vascular endothelial growth factor/vascular permeability factor expression in a mouse model of retinal neovascularization. Proc. Natl. Acad. Sci. USA. 92:905-909.

59. Pierce, E.A., Foley, E.D., and Smith, L.E. 1996. Regulation of vascular endothelial growth factor by oxygen in a model of retinopathy of prematurity [erratum 1997, 115:427]. Arch. Ophthalmol. 114:1219-1228. 